\title{
Photo-enchanced defect reactions in CdS:Ag crystals
}

\author{
L.Yu. Khomenkova, I.V. Markevich \\ Institute of Semiconductor Physics, NAS of Ukraine, 45 prospect Nauky, 03028 Kyiv, Ukraine \\ Phone: (044) 265 7234; fax: (044) 265 8344; e-mail: khomen@lumin.semicond.kiev.ua
}

\begin{abstract}
Two reversible photo-enhanced defect reactions proceeding under visible light illumination have been found in CdS:Ag crystals. The first process leads to the increase of crystal photosensitivity, which has been shown to be caused by creation of "sensitizing" recombination centres. The second process results in photosensitivity degradation that has been proved to be due to disappearance of shallow donors. The initial state of the crystal restores under heating in dark. Both reactions have been shown to vanish after Ag extraction from the crystal. Metastable clusters including $\mathrm{Ag}$ and $\mathrm{Cd}$ atoms are supposed to be responsible for these reactions.
\end{abstract}

Keywords: defects, photo-enhanced reactions

Paper received 11.05.02; accepted for publication 10.12.02.

\section{Introduction}

Photo-enhanced defect reactions (PEDR's) were observed in various semiconductors ( $\mathrm{Si}$, II-VI and III-V compounds) and studied intensively for last several decades [1-3]. The steady interest to these processes is because of they play the main role in degradation of light-emitting devices and photoresistors $[1,3,4]$. On the other hand, PEDR's that cause considerable changes in material conductivity, photosensitivity and emission intensity can be used for registration of information. To control these useful and undesirable effects, the mechanisms of reactions and the nature of defects responsible for these processes have to be elucidated.

Various PEDR's can take place in the same semiconductor depending on the nature and density of native and impurity defects. A number of PEDR's were observed in CdS crystals [2,4-7]. It was shown that these reactions were induced by the change of Coulomb interaction between point defects or complex centre components due to their recharge as a result of photocarrier capture; after such recharge diffusional dissociation of the complex centre or association of point defects became possible [46]. These processes were earlier investigated in nominally undoped and doped with $\mathrm{Cu}$ and $\mathrm{Li} \mathrm{CdS}$ crystals. Recently we found such defect reactions in CdS:Ag crystals [8]. The results of investigation of these reactions and their comparison with those obtained earlier for $\mathrm{CdS}: \mathrm{Cu}$ crystals are adduced below.

\section{Experimental procedure and results}

Bulk CdS crystals grown by sublimation from vapour phase were used. Silver was introduced either under growing or by diffusion from the surface at $800{ }^{\circ} \mathrm{C}$. Bright brown high-resistivity $\left(\rho>10^{9} \Omega \cdot \mathrm{cm}\right)$ crystals were obtained. The crystals were cleft into rectangular parallelepipeds of typical dimensions $5 \times 2 \times 1 \mathrm{~mm}$. The samples were supplied with melted In electrodes.

To reveal photo-enhanced processes the sample was heated up to $500-520 \mathrm{~K}$, cooled in dark to $90 \mathrm{~K}$, and then its photocurrent (PC) and photoluminescence (PL) spectra were measured; next the sample was illuminated with visible light at a certain temperature $T_{L}$ from 100 $450 \mathrm{~K}$ range, cooled to $90 \mathrm{~K}$, and after that $\mathrm{PC}$ and $\mathrm{PL}$ spectra were measured once again. Comparison of the results obtained after cooling in dark and after illumination at different $T_{L}$ showed that two PEDR's took place in the investigated crystals. One of them occurred at $T_{L}>270 \mathrm{~K}$ and resulted in the increase of photosensitivity ("sensitizing" reaction). The other proceeded at $T_{L}>320 \mathrm{~K}$ and caused photosensitivity degradation ("degradation" reaction). These reactions were independent one from the other. Both reactions were found to be reversible, initial state being restored by heating and subsequent cooling without illumination.

When after cooling in dark from $T=500-520 \mathrm{~K}$ the sample was illuminated at $T_{L}=270-310 \mathrm{~K}$, isothermal rise of photocurrent $I_{p h}$ was observed and then steady 


\section{L.Yu. Khomenkova et al.: Photo-enchanced defect reactions in CdS:Ag crystals}

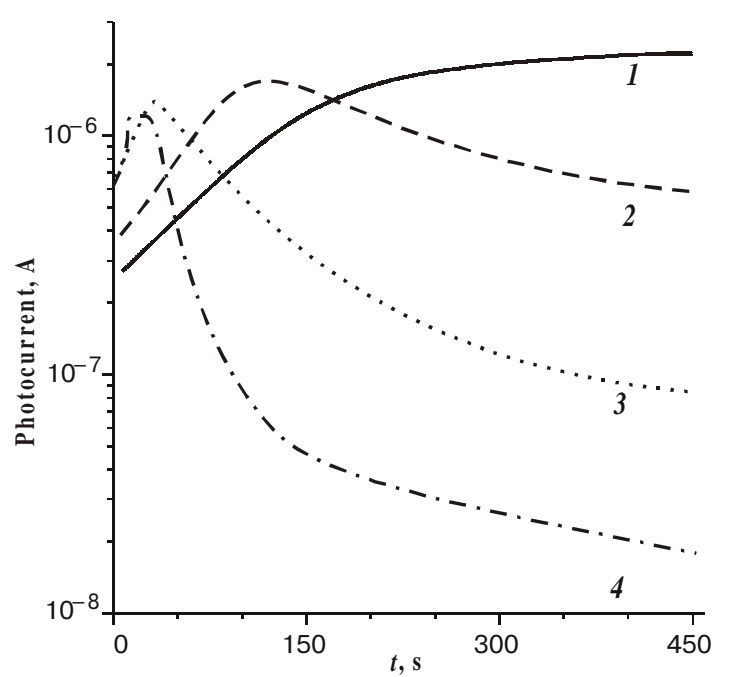

Fig. 1. Photocurrent dependence on time in CdS:Ag crystal after switching on light illumination at 293 (I), 320 (2), 365 (3) and 390 K (4).

value of $I_{p h}$ was reached (Fig.1, curve 1 ). At room temperature this sensitized state retained for a long time in dark, but it could be distracted by heating to $T_{D}>430 \mathrm{~K}$. At $T_{L}>320 \mathrm{~K}$ after initial rise of $I_{p h}$ its subsequent drop occurred under illumination, the higher the temperature the faster the both processes (Fig. 1, curves 2-4). The degradation effect was not influenced by heating up to $450 \mathrm{~K}$ and retired completely only at $500-520 \mathrm{~K}$. At $T_{L}<270 \mathrm{~K}$ the illumination caused no change in crystal photosensitivity. Thus, depending on previous cooling and illumination conditions, the sample at $T<270 \mathrm{~K}$ can be transferred in any of four different states:

A. The initial state (cooling from $500-520 \mathrm{~K}$ in dark);

B. The sensitizing state (illumination at $T_{L}=270$ $310 \mathrm{~K})$;

C. The state with both sensitizing and degradation reactions (illumination at $T_{L}=320-430 \mathrm{~K}$ );

$D$. The state with degradation reaction only (illumination at $T_{L}>430 \mathrm{~K}$ and following cooling in dark).

Each state can be reproduced many times in the same sample.

PC and PL spectra of a typical CdS:Ag crystal in the A-D states are shown in Fig. 2 and Fig. 3 correspondingly. One can see that PEDR's lead to considerable photosensitivity changes in both intrinsic and extrinsic PC maxima. The latter at $\lambda=680-700 \mathrm{~nm}$ is known to be due to excitation of electrons from "sensitizing" recombination centres ( $r$-centres) to c-band ( $r$-centre extrinsic maximum) [9]. In PL spectra, three bands at $\lambda=1.0 \mu \mathrm{m}$ (IR), $\lambda=0.72 \mu \mathrm{m}$ ("red") and $\lambda=0.60 \mu \mathrm{m}$ ("orange") are present. The IR band is known to result from recombination of free electrons on $r$-centres [9]. Two other bands were shown to be caused by recombination of free electrons on "red" and "orange" acceptors [10], the latter being $\mathrm{Ag}_{\mathrm{Cd}}$ centres [8]. The transfer from A to B state leads to considerable increase of IR band intensity. Simultaneously, the decrease of red and orange band intensities takes place, both decreasing equally (Fig. 3,

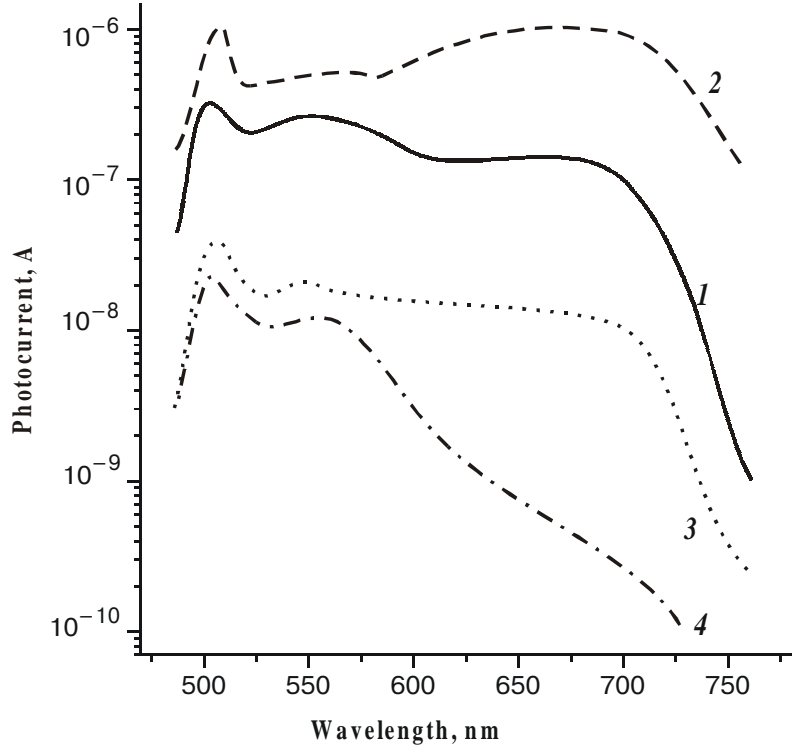

Fig. 2. Photocurrent spectra of CdS:Ag crystal at $90 \mathrm{~K}$ in A (I), B (2), C (3) and D (4) states.

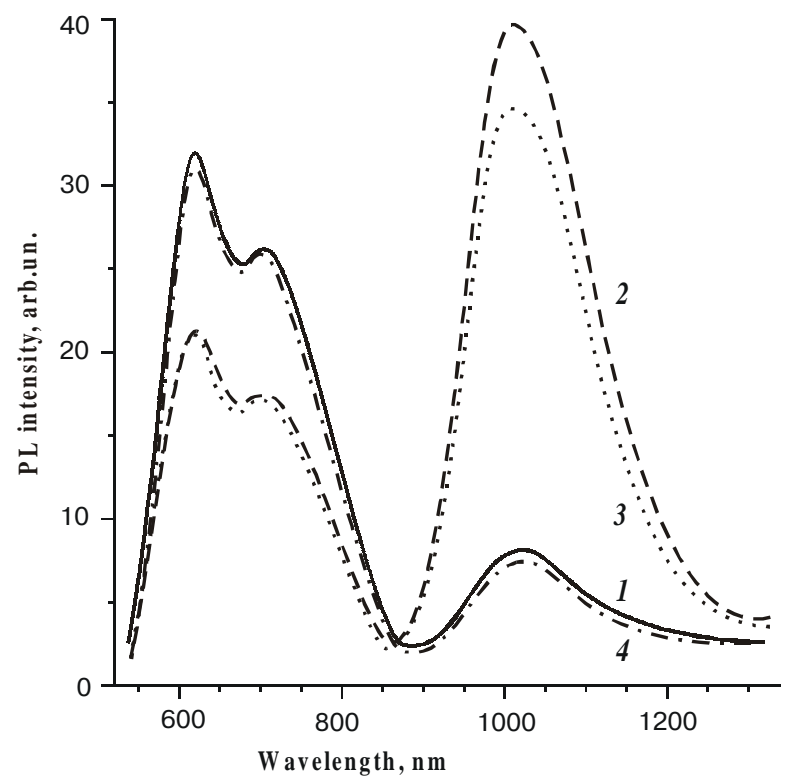

Fig. 3. PL spectra of CdS:Ag crystal at $90 \mathrm{~K}$ in A (1), B (2), C (3) and D (4) states. Excitation light wavelength is $480 \mathrm{~nm}$.

curves 1,2). At the same time, the degradation PEDR causes very slight changes in PL spectra (Fig. 3, curves $3,4)$.

Dark current provided with equilibrium electrons and thermally stimulated current (TSC) were measured in A, $\mathrm{B}$ and $\mathrm{D}$ states in $300-550 \mathrm{~K}$ temperature range (Fig. 4). It was found that equilibrium conductivity did not change as a result of sensitizing PEDR and decreased due to degradation one.

When above measurements had been performed, $\mathrm{Ag}$ was extracted from the samples under external electric field $E_{d}=10^{2} \mathrm{~V} / \mathrm{cm}$ at $T=650 \mathrm{~K}$ (detailed description of the procedure see in [8]). After Ag extraction the crystal acquired bright yellow colour characteristic for undoped 


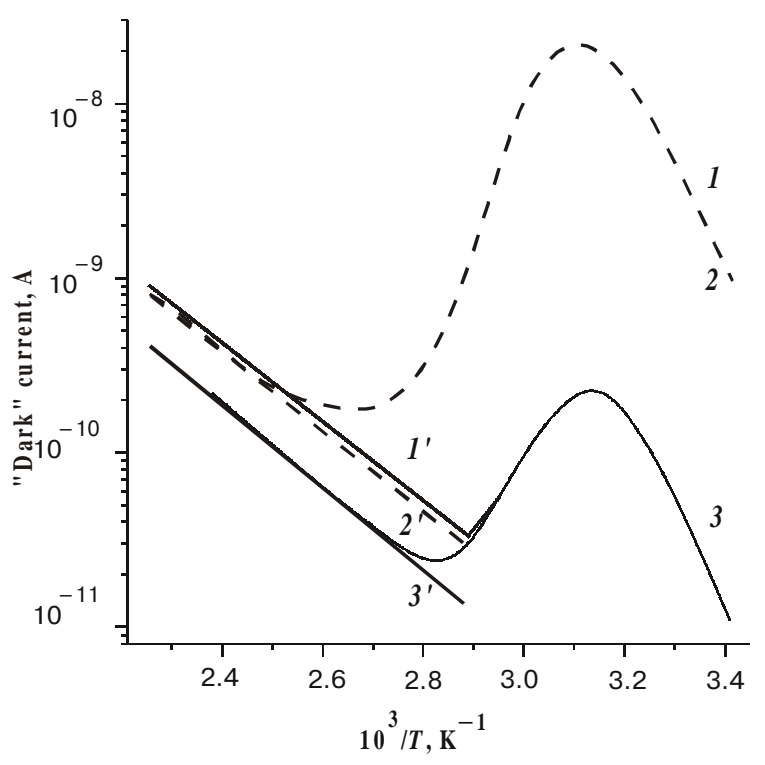

Fig. 4. Temperature dependences of $\operatorname{TSC}(1,2,3)$ and equilibrium conductivity $\left(l^{\prime}, 2^{\prime}, 3^{\prime}\right)$ of CdS:Ag crystal in A $\left(1, l^{\prime}\right)$, B $(2$, $\left.2^{\prime}\right)$ and $\mathrm{D}\left(3,3^{\prime}\right)$ states.

CdS, both investigated PEDR's disappearing. Only a slight sensitizing reaction, that began to proceed at $T_{L}=$ $=330 \mathrm{~K}$ and wholly distracted at $T_{D}=400 \mathrm{~K}$, was observed in "purified" samples (Fig. 5). This PEDR was shown to occur, as a rule, in bulk nominally undoped $\mathrm{CdS}$ crystals and consisted in partial dissociation of $\mathrm{Cd}$ clusters [7].

\section{Discussion}

Earlier investigations showed that lattice defects responsible for PEDR's in CdS were mobile shallow donors, namely cadmium interstitials $\mathrm{Cd}_{\mathrm{i}}[5,6,11,12]$. They are

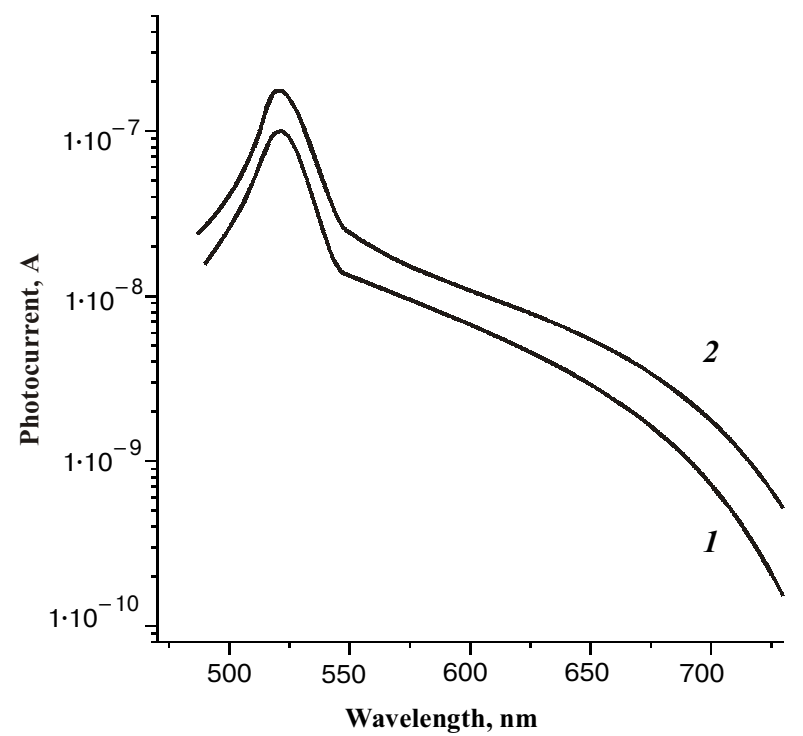

Fig. 5. Photocurrent spectra of CdS:Ag crystal after Ag extraction: 1 - cooling from $520 \mathrm{~K}$ in dark, 2 - illumination at $360 \mathrm{~K}$. always present in $\mathrm{CdS}$ crystals and can associate one with another, as well as with other defects $[5,6,7,11]$. Such associates can be both created and distracted under illumination, which results in the decrease $[5,6,11,12]$ or increase $[2,5-7]$ of shallow donor density and densities of other defects, particularly, $r$-centres $[2,6]$. One can expect that these donors participate also in PEDR's in CdS:Ag crystals.

The change of photocurrent in $r$-centre maximum $I_{p h}^{r}$ means that investigated PEDR's cause the change in the density of electrons on $r$-centres $n_{r}$ under illumination. Really, $I_{p h}^{r} \sim n_{r} \tau_{r}$ [10], where $\tau_{r}=1 / C_{r}^{n} p_{r}$ is free electron lifetime, $C_{r}^{n}$ is the coefficient of electron capture by $r$-centre, $p_{r}=\left(p_{r}^{0}+p_{r}^{\prime}\right)$ is the density of holes on $r$-centres, $p_{r}^{0}$ are holes corresponding to electrons captured by electron traps and $p_{r}^{\prime}$ are holes corresponding to free electrons. Two processes can lead to the increase (decrease) of $n_{r}$ : i) appearance (disappearance) of shallow donors, while the density of $r$-centres remains unchanged; ii) simultaneous appearance (disappearance) of shallow donors and $r$-centres which compensate each other. The first process will result in the increase (decrease) of equilibrium conductivity as well as $\tau_{r}$ due to the decrease (increase) of $p_{r}^{0}$. The second process will not influence on equilibrium conductivity and $\tau_{r}$, and the change of $I_{p h}^{r}$ will be caused only by the change of $n_{r}$ due to creation of new $r$-centres. As Fig. 4 shows, after sensitizing PEDR equilibrium conductivity and TSC values do not change. Since the latter is proportional to $\tau_{r}$ [13] it means that $\tau_{r}$ remains unchanged too. Therefore, one can conclude that the rise of $I_{p h}^{r}$ after sensitizing PEDR is due to creation of compensated $r$-centres.

The changes in PL spectra caused by sensitising PEDR can be also accounted for by the increase of $n_{r}$. The intensity of IR band $W_{r} \sim g_{r}$, where $g_{r}=C_{r}^{p} n_{r} /\left(C_{r}^{p} n_{r}+\sum C_{i}^{p} n_{i}\right)$ is the portion of hole recombination flow through $r$-centres, $C_{r}^{p}, C_{i}^{p}$ are coefficients of hole capture by $r$-centre and any other $i$-recombination centre, $n_{i}$ is electron density on $i$-centres [10]. The increase of $n_{r}$ will lead to the enhancing of hole recombination flow through $r$-centres and the weakening of that through other recombination centres, the decrease of each $g_{i}$ value being the same. This is the effect that is observed (Fig. 3, curves 1,2).

After degradation PEDR equilibrium conductivity and TSC values reduce (Fig. 4 , curves $l^{\prime}, 3^{\prime}$ and 1,3 ). Therefore, one can conclude that as a result of this process the density of shallow donors decreases, while that of acceptors does not. The decrease of shallow donor density results in the rise of $p_{r}^{0}$ and, consequently, in the drop of $\tau_{r}$. In this case, however, the decrease of $n_{r}$ slightly affects PL band intensities (Fig. 3, curves 1, 4 and 2, 3). It means that $I_{p h}^{r}$ drop is mainly due to $\tau_{r}$ decrease. Really, $I_{p h}^{r}$ drop (Fig. 2, curves 1,4 ) is almost equal to that of TSC value (Fig. 4, curves 1,3).

A degradation reaction quite similar to described above was earlier observed in CdS:Cu crystals $[5,11,12]$. This reaction that occurred at $T_{L}>250 \mathrm{~K}$ under visible 


\section{L.Yu. Khomenkova et al.: Photo-enchanced defect reactions in CdS:Ag crystals}

light illumination and distracted at $T_{D}>400 \mathrm{~K}$ in dark also resulted in the reduction of $n_{r}$ due to the decrease of density of shallow donors $[11,12]$. So, the mechanisms of degradation reactions in $\mathrm{CdS}: \mathrm{Cu}$ and $\mathrm{CdS}: \mathrm{Ag}$ crystals can be expected to be the same. The donors that participated in the reaction in CdS:Cu crystals were proved to be cadmium interstitials $\mathrm{Cd}_{\mathrm{i}}$ [12], and their disappearance under illumination was shown to be due to formation of clusters containing $\mathrm{Cd}$ atoms [5,6,11]. Thermal ionization of these clusters in dark led to their dissociation and restoration of the initial $\mathrm{Cd}_{\mathrm{i}}$ density $[5,12]$. It remained vague, however, whether impurity atoms participate in this process. Above results on disappearance of degradation reaction after Ag extraction from the sample testify that Ag-related defects do take part in degradation PEDR in CdS:Ag crystals. One can state, however, that such defects are not $\mathrm{Ag}_{\mathrm{Cd}}$ acceptors responsible for orange band $\lambda_{\max }=600 \mathrm{~nm}$ [8], because this band intensity does not change as a result of degradation reaction (Fig. 3, curves 1, 4 and 2,3). They are not $\mathrm{Ag}_{\mathrm{i}}$ donors either, because such donors were not found in $\mathrm{CdS}: \mathrm{Ag}$ crystals [8]. Most probably, the defects under consideration are complexes that act as priming centres and join mobile donors $\mathrm{Cd}_{\mathrm{i}}$ after recharge due to photocarrier capture. One can suppose that above mentioned clusters in $\mathrm{CdS}: \mathrm{Cu}$ crystals include $\mathrm{Cu}$ atoms as well. Really, our last investigations have shown that extraction of $\mathrm{Cu}$ from CdS:Cu crystals leads to disappearance of degradation PEDR, too. The fact that degradation reactions in $\mathrm{CdS}: \mathrm{Cu}$ and CdS:Ag crystals occur and distract in different temperature ranges can be accounted for by different bound energies of defects in corresponding clusters. Since not only degradation PEDR but also sensitizing one has disappeared after Ag extraction, one can conclude that different metastable complex centres containing Ag are present in the investigated crystals.

In conclusion it is necessary to note, that degradation reaction in CdS:Ag crystals is the most high-temperature PEDR of all observed up to now in CdS. One can think that this reaction can be used for high temperature visible light signal recording.

\section{Conclusion}

Two reversible defect reactions induced by visible light illumination were found in CdS crystals doped with Ag. The first reaction occurred at $T>270 \mathrm{~K}$ and distracted at $T>430 \mathrm{~K}$; this reaction caused the increase of crystal photosensitivity and was shown to consist in creation of "sensitizing" recombination centres. The second reaction occurred at $T>320 \mathrm{~K}$ and distracted at $T>500 \mathrm{~K}$; it led to photosensitivity degradation due to the decrease of free electron lifetime $\tau_{r}$, which was proved to result from disappearance of shallow donors. Each reaction separately and both reactions simultaneously could be proceeded in the same crystal.

It was found that after extraction of $\mathrm{Ag}$ from the sample both sensitizing and degradation reactions vanished. This fact indicates that different Ag-related defects take part in the reactions. These defects are stated to be metastable clusters including Ag and Cd atoms.

The reaction resulted in photosensitivity degradation is the most high-temperature PEDR of all observed in $\mathrm{CdS}$ up to now. This reaction can be used for visible light signal recording in 320-450 temperature range.

\section{References}

1. L.C. Kimerling // Sol.St.Commun., 16(1), pp.171-174 (1975).

2. N.E.Korsunskaya, I.V.Markevich, M.K.Seinkman // Phys.stat.sol., 13(1), pp.25-36 (1966).

3. D.V.Lang // Annu.Rev.Mater.Sci., Paolo Alto, Calif., 12, pp.377-400 (1982)

4. N.E.Korsunskaya, I.V.Markevich, B.R.Dzhumaev, L.V.Borkovskaya, M.K.Sheinkman // Semicond.Phys., Quant Electron and Optoelectr., 2(1), pp.42-45 (1999).

5. M.K.Sheinkman, N.E.Korsunskaya, I.V.Markevich, T.V.Torchinskaya // J.Phys.Chem.Sol., 43(5), pp.475-479 (1982).

6. K.E.Korsunskaya, I.V.Markevich, E.P.Shulga // J.Phys.Chem.Sol., 53(3), pp.469-474 (1992).

7. L.V.Borkovskaya, B.R.Dzhumaev, B.Embergenov, N.E.Korsunskaya, I.V.Markevich // Ukr.Phys.Zhurn., 43(10), pp.1295-1299 (1998).

8. L.V.Borkovskaya, B.M.Bulakh, L.Yu.Khomenkova, I.V.Markevich // Semicond.Phys., Quant.Electron and Optoelectr., 4(3), pp.163-167 (2001).

9. R.H.Bube. "Photoconductivity of solids", 558 p., Eds. John Wiley and Sons, Inc. New York-London (1960).

10. V.Ye.Lashkarev, A.V.Lyubchenko, M.K.Sheinkman, "Nonequillibrium Processes in Semiconductors", 264p. , Eds. "Naukova dumka", Kiev (1981).

11. N.E.Korsunskaya, I.V.Markevich, T.V.Torchinskaya, M.K.Sheinkman // Phys.stat.sol.(a), 60(1), pp.565-572 (1980).

12. N.E.Korsunskaya, I.V.Markevich, T.V.Torchinskaya, M.K.Sheinkman // Pisma v Zh.Tech.Phys., 6(2), pp.120-124 (1980).

13. M.K.Sheinkman, N.E.Korsunskaya // Ukr.Phys.Zhurn., 12(12), pp. 2942-2051 (1967). 